\title{
Synthesis and Application of Fish Gelatin for Hydrogels/ Composite Hydrogels: A Review
}

\author{
Yoni Atma ${ }^{1, * \text { iD }}$ \\ 1 Department of Food Science and Technology, Faculty of Bioindustry, Universitas Trilogi, Jakarta, Indonesia \\ * Correspondence: yoniatma@trilogi.ac.id;
}

Scopus Author ID 57194092488

Received: 24.04.2021; Revised: 5.06.2021; Accepted: 8.06.2021; Published: 13.08.2021

\begin{abstract}
Hydrogels are one of the biopolymers that have been applied and have excellent potential to be developed as a raw material in future food technology, biomedicine, and three-dimensional (3D) bioprinting. Even stigmatized that hydrogels are the only source of bioink for 3D bioprinting. Among natural sources, protein-based hydrogels have advantages in the aspects of biocompatibility, biodegradability, tunability, molecular binding ability, and bioactive properties. Gelatin is a natural protein-based biopolymer that offers potential. Besides its advantages as a natural protein-based hydrogel, gelatin is also inexpensive, usually extracted from processing by-products such as skins and bones. Studies also mentioned that gelatin has the tripeptide motif that promotes cell attachment for subsequent cellular processes, like migration, differentiation, and proliferation. However, most gelatin is derived from mammalian sources, while these sources are limited considering socio-religion, cultural, health aspects. Fish gelatin is the most potential source for alternative gelatin. They have uniqueness and viscosity for bio-fabrication and injectable hydrogels. Therefore, this paper will review the hydrogels based on fish gelatin studied in recent years and the last decade. Here also described the stages in the fabrication of fish gelatin hydrogels/hydrogel composites with different co-polymers, composite materials, polymerization methods, and future intended use of obtained fish gelatin hydrogels/composites.
\end{abstract}

Keywords: biomaterial; biopolymer; composite; fish gelatin; fish GelMA.

(C) 2021 by the authors. This article is an open-access article distributed under the terms and conditions of the Creative Commons Attribution (CC BY) license (https://creativecommons.org/licenses/by/4.0/).

\section{Introduction}

Biopolymers are natural polymers found in the tissues of living organisms, especially plants and animals [1]. Biopolymers have been studied and developed extensively in broad fields such as medicine [2], agriculture [3], food [4], filtration technology [5], and even digital technology [6]. Biopolymers have advantages over synthetic polymers, especially in terms of their sustainability and safety considerations [7]. One of the biopolymers that have been applied and have great potential to be developed as a raw material in food technology [8], biomedicine, and three-dimensional (3D) bioprinting is hydrogels [9]. Hydrogels are polymers that have the ability to hold large amounts of water. Hydrogels are a three-dimensional network that is one of the important biomaterials for biomedical applications [10]. And surprisingly, hydrogels are the only source of bioink for 3D bioprinting (even though few papers mentioned cell aggregate/pellet-based bioinks, but they were generally also using hydrogel as a matrix material) [11]. The 3D technology has been applied in generating an extensive range of biofunctional products, including biomaterials for tissue engineering, bio-medicine, [12] and food applications [13]. 
Hydrogel sources are covering natural origins like proteins and polysaccharides and synthetic hydrogels like poly(ethylene) glycol (PEG), polycaprolactone (PCL), and polylactic acid (PLA) [14]. Natural hydrogels are obtained by protein-based biopolymers such as collagen, gelatin, elastin, and carbohydrate-based biopolymers such as alginate, chitin, chitosan, cellulose, and etc. For sustainability and safety considerations, hydrogels based on natural sources are preferable. For example, alginate, collagen, and gelatin have been used for biomaterial-based bioink production. In addition, among natural sources, protein-based hydrogels have advantages in the aspects of biocompatibility, biodegradability, tunability, molecular binding ability, and bioactive properties [15].

Gelatin is a natural protein-based biopolymer that offers potential. Besides its advantages as a natural protein-based hydrogel, gelatin is also inexpensive, usually extracted from processing by-products such as skins and bones. Recent studies also mention that gelatin has the tripeptide motif formed by arginine-glycine-aspartic acid (Arg-Gly-Asp) sequences or called RGD adhesive motif that promotes cell attachment for subsequent cellular processes, like migration, differentiation, and proliferation [16]. However, most gelatin is derived from mammalian sources, while these sources are limited considering socio-religion and cultural aspects. Therefore, numerous publications and research are seeking an alternative source of gelatin. One of the potential sources of gelatin comes from fish processing by-products. Gelatin from fish processing by-products is mostly derived from skin and bone as well [17]. Until now, studies on fish-based gelatin utilization have been developed rapidly. The research about fish gelatin has been concerned with fish gelatin sources, extraction and characterization $[18,19]$, fish gelatin as biodegradable film and food packaging [20, 21], and fish gelatin as bioactive peptides [22, 23].

Nevertheless, there are limited studies about fish gelatin as a hydrogel. Though the role of the hydrogel is wide, as mentioned above. In addition, the fish gelatin has a uniqueness and specificity as a hydrogel material, such as having a low melting temperature even at $10{ }^{\circ} \mathrm{C}$ which make fish skin gelatin is still in liquid form, and this characteristic will offer the benefits compared to mammalian gelatin for bio-fabricated applications such as in microfluidic droplet generation [24]. Fish gelatin also has a lower viscosity than traditional gelatin. It has been noted that the viscosity of an injectable hydrogel should be low adequately before gelation in order to permit a homogeneous dispersion with encapsulated therapeutic agents [16]. Therefore, this paper will review the hydrogels based on fish gelatin that has been studied in the recent year and last decade. Natural-based hydrogels, including fish gelatin hydrogels, have low mechanical properties in the sole form [25], so they are usually modified and composited with other polymers or materials, so this paper has been discussed for fish gelatin composite hydrogels too. Moreover, here also will describe the stages in the fabrication of fish gelatin hydrogels/hydrogel composites with different co-polymers, composite materials, polymerization methods, and future intended use of fish gelatin hydrogels/composites obtained. All of these aim to provide the researchers concerned about fish gelatin, particularly fish gelatin hydrogel, regarding information, knowledge, research methods and results, and research summary about fish gelatin, especially about fish gelatin as hydrogels/composite hydrogels trends by quickly and completely ways through succinct insights.

\section{Fish Gelatin based Hydrogels/Hydrogel Composites}

Fish gelatin is a promising source of gelatin to develop various natural-based polymers such as basic material for hydrogel fabrications. Fish gelatin-based hydrogels are derived from 
fish gelatin in sole form or modified in their functional group to synthesize the fish gelatinhybrid hydrogels. In comparison, the fish gelatin composite hydrogels are the hydrogels from fish gelatin which are composite with one or more other polymers or materials. The modification of fish gelatin is headed to fabricate a network precursor more easily cross-linked and polymerized. Fish gelatin that has been studied for hydrogels and composite hydrogels fabrication mostly derived from cold-water fish such as the skin of Cod, Pollock, Haddock [26], Sea Bream scales [27], and some of the cold-water fish that have been commercialized for research purposes [24, 28]. In addition, there is also research about fish gelatin hydrogels/composite hydrogels from warm water fish such as Nila Tilapia [29]. These gelatin are usually modified through co-polymer hybridization like methacrylic anhydride [24, 26, 28, 30], glycidyl methacrylate [27, 31], 3-(4-hydroxyphenyl) propionic acid (HPA) [16], and polyacrylamide [32]. Moreover, the composite polymers or materials that have been used including poly(vinyl alcohol) (PVA) [33], mineral nanofibers [27], nano-apatite [32], nanodiamond particles (NDPs) [34], poly(3,4-ethylenedioxythiophene) (PEDOT), polystyrenesulfonate (PSS) [30], and the natural compound like konjac powder [35], as well as other natural polymers like carboxymethyl cellulose (CMC) [24], agar [36], and chitosan [37].

Among those abovementioned materials, either co-polymer or composite materials, methacrylic anhydrate is generally acrylate with fish gelatin to produce fish gelatinmethacrylate (fish GelMA) hydrogels. This co-polymer is most likely because there were many studies concerning methacrylic hybridization with traditional mammalian gelatin to synthesize the hydrogel's acceptable physical or mechanical properties. Even mammalian gelatin methacrylic (GelMA) has been commercialized as one of the best bioinks for 3D printing applications [38]. The co-polymer generally added to produce fish gelatin-based hydrogel is in the form of one block co-synthetic polymer. This co-polymer is attached to be functionalized the functional groups of gelatin to provide better network precursors that are easily cross-linked or polymerized in the further steps. For example, the amine groups in the primary structure of the gelatin acrylate with methacrylate to form GelMA, which has more branches, thus comfortably for cross-linking and polymerization processes. This mechanism is also known as biopolymer functionalization [11], and fish gelatin, in this case, serves as the hydrogel framework.

Research on hydrogels and hydrogel composites based on fish gelatin has various backgrounds with regard to the reasons for choosing fish gelatin. Generally, the fish gelatin is chosen considering some aspects especially social, religious and cultural aspects $[24,26,28$, 36], health risk aspects [27, 28, 39], economical aspects [16, 24, 27, 28, 34], and physicochemical characteristics aspect of fish gelatin [16, 26, 31, 33, 34]. For the socioreligious-cultural aspect, fish gelatin is universally accepted by almost all groups, communities, and religions, while mammalian gelatin is prohibited by certain social, religious, and cultural communities. For instance, porcine gelatin is not permitted for consumption by Muslims and Jewish, while the majority of the Hindu community prohibits beef gelatin. For the health risk aspect, the source of fish gelatin is not risky for spreading diseases (zoonoses) and viruses. Then in the economic aspect, fish gelatin is cheaper because most of the raw material for fish gelatin is produced from the high portion by-products of fish processing such as skin and bones. Furthermore, for the aspect of physicochemical characteristics, fish gelatin has a lower hydrophobic amino acid composition than mammalian gelatin or other natural protein-based hydrogels, so that fish gelatin will remain liquid and viscous at room temperature. This characteristic is required especially when developing 3D bioprinting ink and injection 
technology [26]. Apart from the four aspects mentioned, another reason to promote fish gelatinbased hydrogels is that there are no or very few studies that utilize and develop hydrogels or composite hydrogels by using fish gelatin [29].

\section{Fish Gelatin based Hydrogels/Hydrogel Composites Preparation and Synthesis}

Fish gelatin-based hydrogel preparation and synthesis are mostly carried out by modifying the gelatin structure. This modification is carried out chemically with synthetic copolymers as precursor networks. The stages include fish gelatin preparation, the fish gelatin and co-polymer reaction, defoaming, dialysis, and cross-linking and polymerization [24, 27 , $28,31]$. However, some fish gelatin hydrogels are not modified. After the preparation stage, the fish gelatin hydrogels that only use fish gelatin (sole or without co-polymer), the gelatin is immediately cross-linked. Even the hydrogels from fish gelatin and agar, after being mixed, cross-linking and polymerization are only carried out by heating and cooling approaches [36]. Fish gelatin hydrogels/composite hydrogels building blocks, also their cross-linking and polymerization methods are presented in Table 1.

The initial stage of chemical modification of fish gelatin in the preparation of fish gelatin is carried out by dissolving fish gelatin using polar or polar aprotic solvents at the certain temperature range of $45-60^{\circ} \mathrm{C}[28,30,31,39]$, however, there is also a method that dissolves of gelatin at room temperature as mentioned by Thi et al. (2020) when preparing hydrogels from cold-water fish gelatin which conjugated with 3-(4-hydroxyphenyl) propionic acid (HPA) [16]. The polar solvents that have been used in dissolving of fish gelatin covering deionized water [29, 36, 39], distilled water [35], phosphate-buffered saline (PBS) [28, 30], and sodium carbonate-bicarbonate [24], while an aprotic polar solvent is dimethyl sulfoxide [31]. After the gelatin is dissolved, then the co-polymers is added. The addition of co-polymers is by mixing co-polymer solution in a certain concentration with the fish gelatin solution. Again there was an exception also that requiring special treatment to activate the co-polymer as described by Thi et al. (2020) who activated the co-polymer 3- (4-hydroxyphenyl) propionic acid (HPA) by using 1-ethyl-3- (3- dimethylaminopropyl) -carbodiimide (EDC), and N-hydroxysuccinimide (NHS) in co-solvent deionized water and dimethylformamide (DMF) for 1 hour [16]. In the next step, the mixture of fish gelatin and the co-polymer are homogenized and reacted at $50{ }^{\circ} \mathrm{C}$ for 2-3 hours. However, some reacted the mixtures in a longer time, such as a mixture of fish gelatin with 3- (4-hydroxyphenyl) propionic acid (HPA), which is reacted for 1 day [16] and a mixture of fish gelatin with 4- (dimethylamino) pyridine and glycidyl methacrylate for 2 days [31].

After the fish gelatin and co-polymers had reacted, they were followed by the defoaming or sonication stage. The defoaming stage is carried out to remove air bubbles that are trapped in the mixture of gelatin and co-polymer solution. This stage is performed by using ultrasonic waves at a frequency of $30 \mathrm{kHz}$ for 20-30 min [35]. However, several studies did not carry out the defoaming stage. They immediately carried out the dialysis step of the solution mixture after being reacted [26, 28, 31]. The dialysis stage is carried out by inserting the solution mixture into a tube that has pores (molecular weight cut off/MWCO) of $12-14 \mathrm{kDa}$ then dialysis is carried out against distilled or deionized water for $2-7$ days at $40{ }^{\circ} \mathrm{C}$ to remove the remaining unreacted material and low molecular weight impurities such as unreacted methacrylate or methacrylic acid which are potentially cytotoxic during the acrylation process with the amine group of protein gelatin. The dialysis stage is the purification hydrogels by washing monomers or remaining photoinitiators (which are sometimes added early) to reduce 
toxicity $[24,26,28]$. In the dialysis stage, distilled or deionized water is usually changed every one to two days.

Table 1. The network precursors, composite materials, and methods of cross-linking and polymerization of fish gelatin hydrogels/composite hydrogels.

\begin{tabular}{|c|c|c|c|c|}
\hline Co-polymer & $\begin{array}{l}\text { Network } \\
\text { precussor }\end{array}$ & Composite materials & $\begin{array}{c}\text { Method of } \\
\text { crosslinking and } \\
\text { polimerization }\end{array}$ & References \\
\hline $\begin{array}{l}\text { 3-(4- } \\
\text { hydroxyphenyl) } \\
\text { propionic acid } \\
\text { (HPA) }\end{array}$ & Fish GelHPA & None & UV crosslinking & {$[16]$} \\
\hline $\begin{array}{l}\text { methacrylic } \\
\text { anhydride }\end{array}$ & Fish GelMA & $\begin{array}{l}\text { CMC, PEGylated } \\
\text { Liposomes }\end{array}$ & UV crosslinking & {$[24]$} \\
\hline $\begin{array}{l}\text { Methacrylic } \\
\text { anhydride }\end{array}$ & Fish GelMA & None & UV crosslinking & {$[26]$} \\
\hline $\begin{array}{l}\text { Glycidyl } \\
\text { methacrylate }\end{array}$ & $\begin{array}{l}\text { Fish gelatin- } \\
\text { Methacrylate } \\
\text { (fish GelMA) }\end{array}$ & Imogolite nanofibers & UV crosslinking & {$[27]$} \\
\hline $\begin{array}{l}\text { Methacrylic } \\
\text { anhydride } \\
\text { (MA) }\end{array}$ & Fish GelMA & None & UV crosslinking & {$[28]$} \\
\hline $\begin{array}{l}\text { Methacrylic } \\
\text { anhydride }\end{array}$ & Fish GelMA & $\begin{array}{l}\text { poly }(3,4- \\
\text { ethylenedioxythiophene) } \\
\text { :polystyrenesulfonate } \\
\text { (PEDOT:PSS) }\end{array}$ & UV crosslinking & {$[30]$} \\
\hline $\begin{array}{l}\text { Glycidyl } \\
\text { methacrylate }\end{array}$ & Fish GelMA & None & Photocrosslinking & {$[31]$} \\
\hline Polyacrilamide & $\begin{array}{l}\text { Fish gelatin- } \\
\text { polyacrilamide } \\
\text { (fish Gel- } \\
\text { PAAm) }\end{array}$ & Nanoapatite & UV crosslinking & {$[32]$} \\
\hline None & Fish gelatin & $\begin{array}{l}\text { Polyvinyl alcohol } \\
\text { (PVA) }\end{array}$ & $\begin{array}{l}\text { Physical } \\
\text { crosslinking }\end{array}$ & [33] \\
\hline None & Fish gelatin & $\begin{array}{l}\text { Nanodiamond particles } \\
\text { (NDPs) }\end{array}$ & $\begin{array}{l}\text { Enzymatic } \\
\text { crosslinking }\end{array}$ & {$[34]$} \\
\hline None & Fish gelatin & Konjac powder & $\begin{array}{l}\text { Physical } \\
\text { crosslinking }\end{array}$ & {$[35]$} \\
\hline None & Fish gelatin & Agar & $\begin{array}{l}\text { Physical } \\
\text { crosslinking }\end{array}$ & {$[36]$} \\
\hline None & Fish gelatin & None & $\begin{array}{l}\text { Enzymatic } \\
\text { crosslinking }\end{array}$ & [39] \\
\hline
\end{tabular}

The cross-linking and polymerization stages are the final and critical stages in modified hydrogel synthesis. The cross-linking and polymerization stages of fish-based hydrogels can be carried out using photo-crosslinking, enzymatic cross-linking, and physical cross-linking approaches. In photo-crosslinking, fish gelatin and or polymer mixture is irradiated at ultraviolet (UV) wavelengths with the addition of photoinitiator radicals such as 2-hydroxy-1(4- (hydroxyethoxy) phenyl) -2-methyl-1-propanone (Irgacure 2959) as much as 0.1-0.5\% at temperature $80{ }^{\circ} \mathrm{C}[27,28,32]$. Meanwhile, cross-linking enzymatic is carried out by adding glutaraldehyde or transglutaminase and then mixed slowly to prevent bubbles again. Incubation, allowing the enzymatic cross-linking process for 8 hours at $37{ }^{\circ} \mathrm{C}$ [34], and then immediately cooled at room temperature or $25{ }^{\circ} \mathrm{C}$. Furthermore, physical cross-linking is carried out by heating at $60{ }^{\circ} \mathrm{C}$, cooling with water, neutralization by washing several times using buffers, and storing at $4{ }^{\circ} \mathrm{C}$ for approximately $24 \mathrm{~h}$ to strengthen the gel [35]. After the cross-linking and polymerization stages, the modified fish gelatin hydrogels will be obtained, such as a chemically modified fish gelatin-methacrylic hydrogel (fish GelMA) where the name and abbreviation are depending on the name of conjugated co-polymer. 
In composite form, fish gelatin-based hydrogels are fabricated from 1) fish, gelatin-copolymer hydrogels such as fish gelatin-methacrylate hydrogels (fish GelMA) and 2,) from fish gelatin alone without modifications which both of them called pre-polymer. Research by Spancer et al. (2018) synthesized composite hydrogels by mixing fish GelMA with poly $(3,4-$ ethylenedioxythiophene) (PEDOT): polystyrene sulfonate (PSS). Fish GelMA preparation was carried out by dissolving the pre-polymer in $7 \%(\mathrm{~m} / \mathrm{v})$ distilled water. While PEDOT: PSS solution is sterilized and filtered to remove large aggregate, then sonicated to dissolving and dispersing the composite particles in the pre-polymer solution before finally being cross-linked [30]. In the study conducted by Liu et al. (2020), a 7\% carboxymethyl cellulose (CMC) was mixed with fish GelMA pre-polymer for the synthesis of hydrogel composites [24]. And Serafim et al. (2016) used a pre-polymer fish gelatin-polyacrylamide (fish Gel-PAAm), then added mineral nano-apatite before being cross-linked to form a fish gelatin nanocomposite hydrogel [32]. Meanwhile, the synthesis of hydrogel composites using fish gelatin without modification as a pre-polymer has been carried out by mixing the composite materials into the fish gelatin solution, then cross-linked and polymerized. Composite materials that have been mixed to form fish gelatin composite hydrogels, including polyvinyl alcohol (PVA) [33], nanodiamond particles (NDPs) [34], konjac powder [35], agar [36], and chitosan [37]. Besides these two approaches, there was also research in which the composite materials mixed early into reacted fish gelatin-co-polymer solution, followed by cross-linking and polymerization, as mentioned by Teramoto et al. (2012), which directly added imogolite nanofibers during the preparation of fish gelatin and co-polymer. After that, the usually modified hydrogel synthesis stages (defoaming/sonication, dialysis) are performed. Indeed, on this occasion, it should be ensured that composite materials remain unchanged during the sonication process [27].

\section{Fish Gelatin based Hydrogels/Hydrogel Composites Applications}

Predominantly, research on fish gelatin hydrogels/hydrogel composites is intended for drug delivery, tissue engineering, and wound dressing. While, there was also research that specifically developed fish gelatin-based hydrogels to facilitate its fabrication, such as injectable bioink or bioink for 3D printing. Research conducted by Thi et al. (2020) about the conjugation of fish gelatin with 3-(4-hydroxyphenyl) propionic acid (HPA) to form a fish GelHPA hydrogel shown that the fish Gel-HPA hydrogel has an elastic modulus that can be used for a wide range of biomedical applications. The fish Gel-HPA is also used as 2D/3D biomaterials containing human dermal fibroblasts (hDFBs) cells, and it concluded that the cells spread higher on fish Gel-HPA hydrogels than on mammalian gelatin hydrogels. The fishbased hydrogel matrix is softer, offering favorable conditions for cell growth and migration shown by the cells spread in the form of spindles within. The combination of morphological analysis and cell proliferation showed that fish-based hydrogel has excellent biocompatibility for cellular activities, so it can potentially be an injectable scaffold for cells/ drugs delivery in regenerative tissue medicine [16]. Then, in the research handled by Wang et al. (2017), fish GelMA was synthesized as bioink for 3D printing. The results showed that fish-based hydrogel had a lower viscosity than mammalian gelatin methacrylate (GelMA) and had less temperature variation regarding viscosity. These will be useful for biofabrication. After all, biofabrication techniques such as inkjet bioprinting only use low viscosity bioinks. The fish GelMA also has a smaller variety of hydrogel droplets due to the influence of temperature and proper generation at $20{ }^{\circ} \mathrm{C}$ correlated with the thermal stability [31]. 
As drug delivery, a study conducted by Liu et al. (2020), which uses fish gelatin composite hydrogel as Doxorubicin (Dox) delivery platform, revealed that 3D fish gelatin composite hydrogel has a controlled drug release behavior. It is based on patch shapes and UV exposure time for cross-linking. While Kang et al. (2019) also use Dox drug shown fish GelMA hydrogel is a hydrogel with $\mathrm{pH}$-dependent sustained release behavior. Approximately $11 \%$ DOX was released slowly at $\mathrm{pH} 4.5$ for $72 \mathrm{~h}$, the drug release rate would be increased by the degradation of fish GelMA in the cells or in vivo environments, it is based on staining cells data which was using live/dead cells assay and concluding that fish GelMA is biocompatible. In addition, the Dox coated fish GelMA hydrogel did not show any significant cytotoxicity, while the lower concentration of free Dox showed slight cytotoxicity. Overall, fish GelMA is a potential carrier agent of small-molecule drugs, and their release behavior under acidic conditions could be worth for intracellular delivery of drugs [26]. Furthermore, the study by Rattana et al. (2015) showed that fish gelatin can also be used as a hydrogel for controlled drug delivery of salicylic acid and 5-sulfosalicylic acid. These drugs have similar interactions with both fish gelatin hydrogel and mammalian gelatin hydrogel [39]. Nowadays, studies about the drug delivery performance of natural hydrogels have been increased dramatically [40, 41].

For tissue engineering applications, a study by Spancer et al. (2018) synthesized fish GelMA composed with poly(3,4-ethylenedioxythiophene):polystyrene sulfonate (PEDOT:PSS) hydrogel. The result showed that the composite hydrogel was stable under physiological conditions and supported the viability and spread of $\mathrm{C} 2 \mathrm{C} 12$ myoblasts. This study also indicates that soft and flexible fish-based hydrogels coupled with their conductivity are enabled on interfacing with human tissues such as skin or neural probes [30]. Another study conducted by Yoon et al. (2016) showed that fish gelatin hydrogel (fish GelMA) has a higher swelling ratio than mammalian gelatin hydrogel and high fidelity for microscale patterning application. The fish gelatin hydrogel has high biocompatibility behavior, where the cells cultured in fish GelMA hydrogel have high viability, proliferation, and formed networks with surrounding cells. In addition, the fish gelatin hydrogel in the form of 3D hydrogel capable encapsulated cells until $24 \mathrm{~h}$ proven by high viability and proliferation of retained cells [28]. The 3D bioprinting technology is emerging to challenge the gaps faced by conventional techniques and boost the implementation of additive manufacturing [42].

As a wound dressing, although many systems such as films, foams, and hydrogels have been expanded, the hydrogels are the supreme nominee because they potentially fulfill the specifications for wound healings [33]. Zhou et al. (2020) fabricated fish gelatin composite hydrogels using konjac glucomannan powder as a composite material and then loaded matrine (an active ingredient of many Chinese herbal medicines) with various concentrations. The result showed that fish gelatin composite hydrogels had great transparency, a smooth surface, excellent swelling capacity, and sufficient water evaporation rate, where these characteristics are favorable for wound dressing applications. The hydrogel exhibited good antibacterial activity both for gram-positive and gram-negative bacteria. It is suggested that the hydrogel coalesced with the composite materials, which have antimicrobial activity. In addition, the composite hydrogels had satisfactory blood compatibility, so they should be considered nonhemolytic materials because their hemolytic values were merely under $2 \%$ [35]. Ren et al. (2020) also fabricated composite hydrogels from fish gelatin and polyvinyl alcohol (PVA) for wound dressing application. This study demonstrated that all composite hydrogels had good formability. The composite hydrogels have a preferable water evaporation rate than uncomposted fish gelatin. It is generally accepted that hydrogels with a low water evaporation 
rate would diminish, replacing times which correlated with fasten healing and less pain [33]. In addition, the loaded salicylic acid into fish gelatin composite hydrogels made hydrogels exhibited fine antimicrobial activity against Staphylococcus aureus and Escherichia coli ATCC 25922. Moreover, the obtained composite hydrogels have distinguished physical properties, including swelling behavior. All of these parameters are required as ideal wound dressing hydrogels [39].

Table 2. Intended applications of fish gelatin hydrogels/composite hydrogels.

\begin{tabular}{|c|c|c|}
\hline Source of gelatin & Applications & Reference \\
\hline Cold-water fish & $\begin{array}{l}\text { Injectable } \\
\text { bioink for } \\
\text { drug delivery } \\
\text { and tissue } \\
\text { engineer }\end{array}$ & [16] \\
\hline Cold-water fish & $\begin{array}{l}\text { Drug } \\
\text { delivery }\end{array}$ & [24] \\
\hline Cod skin & $\begin{array}{l}\begin{array}{l}\text { Drug } \\
\text { delivery }\end{array} \\
\end{array}$ & [26] \\
\hline Haddock skin & $\begin{array}{l}\text { Drug } \\
\text { delivery }\end{array}$ & [26] \\
\hline Pollock skin & $\begin{array}{l}\text { Drug } \\
\text { delivery }\end{array}$ & [26] \\
\hline Cold-water fish & $\begin{array}{l}\text { Tissue } \\
\text { engineering }\end{array}$ & [28] \\
\hline Cold-water fish & $\begin{array}{l}\text { Biomaterial } \\
\text { (tissue } \\
\text { engineering) }\end{array}$ & [30] \\
\hline Cold-water fish & $\begin{array}{l}\text { Bioink for } \\
\text { 3D printing }\end{array}$ & [31] \\
\hline Fish gelatin* & $\begin{array}{l}\text { Wound } \\
\text { dressing }\end{array}$ & [33] \\
\hline Fish gelatin* & $\begin{array}{l}\text { Wound } \\
\text { dressing }\end{array}$ & [35] \\
\hline Cold-water fish & $\begin{array}{l}\text { Drug } \\
\text { delivery }\end{array}$ & [39] \\
\hline
\end{tabular}

* not mentioned details

\section{Conclusions}

Fish gelatin hydrogels/composite hydrogels preparation and synthesis are mostly carried out by modifying the gelatin structure first. This modification is usually carried out by chemical approaches with the addition of synthetic co-polymers so that the hydrogel is easily cross-linked and polymerized. Methacrylic anhydrate is generally acrylate with fish gelatin to produce fish gelatin-methacrylate (fish GelMA) hydrogels. At the same time, the fish gelatin composite hydrogels are fabricated by fish gelatin or fish gelatin-copolymer as precursor networks. The composite polymers or materials that have been blended into fish gelatin including poly(vinyl alcohol) (PVA), mineral nanofibers, nano-apatite, nanodiamond particles (NDPs), poly(3,4-ethylenedioxythiophene) (PEDOT), poly-styrenesulfonate (PSS), and the natural compound like konjac powder, as well as other natural polymers like carboxymethyl cellulose (CMC), agar, and chitosan. Then, mainly, research on fish gelatin hydrogels/hydrogel composites is intended for drug delivery, tissue engineering, and wound dressing. While, there is also research that specifically developed fish gelatin-based hydrogels to facilitate its fabrication, such as injectable bioink and bioink for 3D printing. 


\section{Funding}

This research received no external funding.

\section{Acknowledgments}

This research has no acknowledgment.

\section{Conflicts of Interest}

The authors declare no conflict of interest.

\section{References}

1. Yadav, P.; Yadav, H.; Shah, V.G.; Shah, G.; Dhaka, G. Biomedical biopolymers, their origin and evolution in biomedical sciences: A systematic review. Journal of Clinical and Diagnostic Research 2015, 9, ZE21ZE25, https://doi.org/10.7860/JCDR/2015/13907.6565.

2. Wróblewska-Krepsztul, J.; Rydzkowski, T.; Michalska-Pozoga, I.; Thakur, V.K. Biopolymers for biomedical and pharmaceutical applications: Recent advances and overview of alginate electrospinning. Nanomaterials 2019, 9, 404, https://doi.org/10.3390/nano9030404.

3. Tomadoni, B.; Casalongué, C.; Alvarez, V.A. Biopolymer-based hydrogels for agriculture applications: swelling behavior and slow release of agrochemicals. In: Gutiérrez, T. (ed.) Polymers for Agri-Food Applications 2019, 99-125, Springer, Cham., https://doi.org/10.1007/978-3-030-19416-1_7.

4. Wicochea-Rodríguez, J.D; Chalier, P.; Ruiz, T.; Gastaldi, E. Active food packaging based on biopolymers and aroma compounds: How to design and control the release. Frontiers Chemistry 2019, 7, 398, https://doi.org/10.3389/fchem.2019.00398.

5. Shaikhiev, I.G.; Kraysman, N.V.; Sverguzova, S.V.; Spesivtseva, S.E.; Yarothckina, A.N. Fish scales as a biosorbent of pollutants from wastewaters and natural waters (a literature review). Biointerface Research in Applied Chemistry 2020, 10, 6893 - 6905, https://doi.org/10.33263/BRIAC106.68936905.

6. Li, X.; Ding, C.; Li, X.; Yang, H.; Liu, S.; Wang, X.; Zhang, L.; Sun, Q.; Liu, X.; Chen, J. Electronic biopolymers: From molecular engineering to functional devices. Chemical Engineering Journal 2020, 397 , https://doi.org/10.1016/j.cej.2020.125499.

7. Gowthaman, N.S.K.; Lim, H.N.; Sreeraj, T.R.; Amalraj, A.; Gopi, S. Advantages of biopolymers over synthetic polymers: social, economic, and environmental aspects. In: Thomas, S.; Gopi, S.; Amalraj, A. (eds.) Biopolymers and their Industrial Applications 2020, 351-372, Elsevier, https://doi.org/10.1016/C2018-005189-0.

8. Klein, M.; Poverenov, E. Natural biopolymer-based hydrogels for use in food and agriculture. Journal of The Science of Food and Agriculture 2020, 100, 2337-2347, https://doi.org/10.1002/jsfa.10274.

9. Zhang, Y.; Zhou, D.; Chen, J.; Zhang, X.; Li, X.; Zhao, W.; Xu, T. Biomaterials Based on Marine Resources for 3D Bioprinting Applications. Marine Drugs 2019, 17, 555, https://doi.org/10.3390/md17100555.

10. Khalil, A.M. Interpenetrating polymeric hydrogels as favorable materials for hygienic applications. Biointerface Research in Applied Chemistry 2020, 10, 5011-5020, https://doi.org/10.33263/BRIAC102.011020.

11. Valot, L.; Martinez, J.; Mehdi, A.; Subra, G. Chemical insights into bioinks for 3D printing. Chemical Society Reviews 2019, 48, 4049-4086, https://doi.org/10.1039/c7cs00718c.

12. Murphy, S. V.; Atala, A. 3D bioprinting of tissues and organs. Nature Biotechnology 2014, 32, 773-785, https://doi.org/10.1038/nbt.2958.

13. Liu, Z.; Zhang, M. 3D food printing technologies and factors affecting printing precision. In: Godoi, F.C.; Bhandari, B.R.; Prakash, S.; Zhang, M. (eds.), Fundamentals of 3D Food Printing and Applications 2019, 19-40, Elsevier Inc, https://doi.org/10.1016/b978-0-12-814564-7.00002-x.

14. Leberfinger, A. N.; Ravnic, D. J.; Dhawan, A.; Ibrahim, T. O. Concise review: bioprinting of stem cells for transplantable tissue fabrication. Stem Cells Transl. Med. 2017, 6, 1940-1948, https://doi.org/10.1002/sctm.17-0148.

15. Panahi, R.; Baghban-Salehi, M. Protein-Based Hydrogels. In: Mondal M. (ed.) Cellulose-Based Superabsorbent Hydrogels, Polymers and Polymeric Composites: A Reference Series 2019, pp 1561-1600, Springer, Cham. https://doi.org/10.1007/978-3-319-77830-3_52.

16. Thi, P.L.; Lee, Y.; Tran, D.L.; Thi, T.T.H.; Park, K.D. Horseradish peroxidase-catalyzed hydrogelation of fish gelatin with tunable mechanical properties and biocompatibility. Journal of Biomaterials Applications 2020, https://doi.org/10.1177/0885328219899787. 
17. Karayannakidis, P. D.; Zotos, A. Fish processing by-products as a potential source of gelatin: A review. Journal of Aquatic Food Product Technology 2016, 25, 65-92, http://doi.org/10.1080/10498850.2013.827767.

18. Atma, Y.; Ramdhani, H.; Mustopa, A.Z.; Pertiwi, M.; Maisarah R. Karakteristik fisikokimia gelatin tulang ikan patin (Pangasius sutchi) hasil ekstraksi menggunakan limbah buah nanas (Ananas comosus). Agritech 2018, 38, 56, https://doi.org/10.22146/agritech.29821.

19. da Trindade Alfaro, A.; Balbinot, E.; Weber, C.I.; Tonial, I.B.; Machado-Lunkes, A. Fish gelatin: characteristics, functional properties, applications and future potentials. Food Engineering Reviews 2015, 7, 33-44, https://doi.org/10.1007/s12393-014-9096-5.

20. Gómez-Guillén, M.C.; Pérez-Mateos, M.; Gómez-Estaca, J.; López-Caballero, E.; Giménez, B.; Montero, P. Fish gelatin: a renewable material for developing active biodegradable films. Trends in Food Science \& Technology 2009, 20, 3-16, https://doi.org/10.1016/j.tifs.2008.10.002.

21. Matche, R.S.; Anup, G.J.; Mrudula, G. Development of biodegradable films from marine ingredients incorporated with natural antimicrobial agents for food packaging. Journal of Packaging Technology and Research 2020, 4, 45-55, https://doi.org/10.1007/s41783-020-00085-X.

22. Atma, Y.; Lioe, H. N.; Prangdimurti, E.; Seftiono, H.; Taufik, M.; Mustopa, A. Z. Dipeptidyl Peptidase IV (DPP-IV) Inhibitory Activity of Ultrafiltration and Gel Filtration Fractions of Gelatin Hydrolysates Derived from Bone of Fish for Antidiabetes. Int J Adv Sci Eng Inf Technol 2019, 9, 2096-2103, https://doi.org/10.18517/ijaseit.9.6.10658.

23. Nasri, M. Bioactive peptides from fish collagen by-products. In: Simpson, B.K.; Aryee, A.N.A.; Toldra, F. (eds), By-products from Agriculture and Fisheries: Adding Value for Food, Feed, Pharma, and Fuels 2019, John Wiley \& Sons Ltd, https://doi.org/10.1002/9781119383956.ch13.

24. Liu, J.; Tagami, T.; Ozeki, T. Fabrication of 3D-printed fish-gelatin-based polymer hydrogel patches for local delivery of PEGylated liposomal doxorubicin. Marine Drugs 2020, 18, 325, https://doi.org/10.3390/md18060325.

25. Xing, Q., Yates, K., Vogt, C., Qian, Z., Frost, M. C., \& Zhao, F. Increasing mechanical strength of gelatin hydrogels by divalent metal ion removal. Scientific Reports 2014, 4, 1-10, https://doi.org/10.1038/srep04706.

26. Kang, M.G.; Lee, M.Y.; Cha, J.M.; Lee, J.K.; Lee, S.C.; Kim, J.; Hwang, Y.; Bae, H. Nanogels derived from fish gelatin: Application to drug delivery system. Marine Drugs 2019, 17, 246, https://doi.org/10.3390/md17040246.

27. Teramoto, N.; Hayashi, A.; Yamanaka, K.; Sakiyama, A.; Nakano, A.; Shibata, M. Preparation and mechanical properties of photo-crosslinked fish gelatin/imogolite nanofiber composite hydrogel. Materials 2012, 5, 2573-2585, https://doi.org/10.3390/ma5122573.

28. Yoon, H.J.; Shin, S.R.; Cha, J.M.; Lee, S.; Kim, J.; Do, J.T.; Song, H.; Bae, H. Cold water fish gelatin methacryloyl hydrogel for tissue engineering application. PLoS One 2016, 11, e0163902, https://doi.org/10.1371/journal.pone.0163902.

29. Chen, H.; Shi, P.; Fan, F.; Chen, H.; Wu, C.; Xu, X.; Wang, Z.; Du, M. Hofmeister effect-assisted one step fabrication of fish gelatin hydrogels. LWT - Food Science and Technology 2020, https://doi.org/10.1016/j.lwt.2019.108973.

30. Spencer, A.R.; Primbetova, A.; Koppes, A.N.; Koppes, R.A.; Fenniri, H.; Annabi, N. Electroconductive gelatin methacryloyl-PEDOT:PSS composite hydrogels: Design, synthesis, and properties. ACS Biomaterials Science and Engineering 2018, 4, 1558-1567, https://doi.org/10.1021/acsbiomaterials.8b00135.

31. Wang, Z.; Tian, Z.; Menard, F.; Kim, K. Comparative study of gelatin methacrylate hydrogels from different sources for biofabrication applications. Biofabrication 2017, 9, 044101, https://doi.org/10.1088/17585090/aa83cf.

32. Serafim, A.; Cecoltan, S.; Vasile, E.; Stancu, I. Polyacrylamide-gelatin hydrogels and mineralized hybrids: influence of gelatin type. U.P.B. Sci. Bull. 2015, 77, 189-200.

33. Ren, T.; Gan, J.; Zhou, L.; Chen, H. Physically cross-linked hydrogels based on poly (vinyl alcohol) and fish gelatin for wound dressing application: Fabrication and characterization. Polymers 2020, 12, 1729, https://doi.org/10.3390/polym12081729.

34. Cecoltan, S.; Serafim, A.; Dragusin, D.M.; Lungu, A.; Lagazzo, A.; Barberis, F.; Stancu, I. The potential of NDPs-loaded fish gelatin fibers as reinforcing agent for fish gelatin hydrogels. Key Engineering Materials 2016, 695, 278-283, https://doi.org/10.4028/www.scientific.net/KEM.695.278.

35. Zhou, L.; Xu, T.; Yan, J.; Li, X.; Xie, Y.; Chen, H. Fabrication and characterization of matrine-loaded konjac glucomannan/ fish gelatin composite hydrogel as antimicrobial wound dressing. Food Hydrocolloids 2020, 104, 105702, https://doi.org/10.1016/j.foodhyd.2020.105702.

36. Somboon, N.; Karrila, T.T.; Kaewmanee, T.; Karrila, S.J. Properties of gels from mixed agar and fish gelatin. International Food Research Journal 2014, 21, 485-492.

37. da Silva, M.A.; Bode, F.; Drake, A.F.; Goldoni, S.; Stevens, M.M.; Dreiss, C.A. Enzymatically cross-linked gelatin/chitosan hydrogels: Tuning gel properties and cellular response. Macromolecular Bioscience 2014, 14, 817-830, https://doi.org/10.1002/mabi.201300472. 
38. Pepelanova, I.; Kruppa, K.; Scheper, T.; Lavrentieva, A. Gelatin-methacryloyl (GelMA) hydrogels with defined degree of functionalization as a versatile toolkit for 3D cell culture and extrusion bioprinting. Bioengineering 2018, 5, 55, https://doi.org/10.3390/bioengineering5030055.

39. Rattana, M.; Paradee, N.; Sirivat, A.; Niamlang, S. Porcine and fish gelatin hydrogels for controlled release of salicylic acid and 5-sulfosalicylic acid. Int. J. Drug Dev. \& Res 2015, 7, 107-117.

40. Ullah, F.; Javed, F.; Zakaria, M.R.; Jamila, N.; Khattak, R.; Khan, A.N.; Aki, H.M. Determining the molecular-weight and interfacial properties of chitosan built nanohydrogel for controlled drug delivery applications. Biointerface Research in Applied Chemistry 2019, 9, $4452 \quad-\quad 4457$, https://doi.org/10.33263/BRIAC96.452457.

41. Ullah, F.; Javed, F.; Khan, A.N.; Kudus, M.H.A.; Jamila, N.; Minhaz, A.; Aki, H.M. Synthesis and surface modification of chitosan built nanohydrogel with antiviral and antimicrobial agent for controlled drug delivery. Biointerface Research in Applied Chemistry 2019, 9, $4439 \quad$ - 4445, https://doi.org/10.33263/BRIAC96.439445.

42. Horst, D.J.; de Andrade Junior, P.P.; Duvoisin, C.A.; de Almeida Vieira, R. Fabrication of conductive filaments for 3d-printing: polymer nanocomposites. Biointerface Research in Applied Chemistry 2020, 10, 6, 6577 - 6586, https://doi.org/10.33263/BRIAC106.65776586. 